\title{
35 ans âpres la disparition des facultés : les universités françaises à l'heure de la coexistence
}

Bruno Ollivier et Olivier Pulvar

\section{(2) OpenEdition}

12 Journals

Édition électronique

URL : http://journals.openedition.org/communicationorganisation/2926

DOI : 10.4000/communicationorganisation.2926

ISSN : $1775-3546$

Éditeur

Presses universitaires de Bordeaux

Édition imprimée

Date de publication : 1 mai 2004

ISSN : 1168-5549

Référence électronique

Bruno Ollivier et Olivier Pulvar, « 35 ans âpres la disparition des facultés : les universités françaises à I'heure de la coexistence », Communication et organisation [En ligne], 24 | 2004, mis en ligne le 27 mars 2012, consulté le 19 avril 2019. URL : http://journals.openedition.org/

communicationorganisation/2926 ; DOI : 10.4000/communicationorganisation.2926

Ce document a été généré automatiquement le 19 avril 2019

(c) Presses universitaires de Bordeaux 


\title{
35 ans âpres la disparition des facultés : les universités françaises à l'heure de la coexistence
}

\author{
Bruno Ollivier et Olivier Pulvar
}

\section{Des identités multiples et en mutation.}

1 Comme tous les acteurs sociaux d'une organisation, l'action des membres de la communauté universitaire dépend des choix identitaires qui leur font déterminer leurs intérêts. On entend ici identité au sens d'un ensemble de représentations qui définit pour chacun ce qu'il estime avoir en commun avec les autres, qui l'ait naître un sentiment d'appartenance, oriente les représentations de soi et des autres et l'action qu'il a dans l'organisation. Chaque acteur est virtuellement porteur d'une grande quantité d'identités, et choisit, en fonction de ses intérêts et de sa stratégie, de mettre en avant telle ou telle identité dans telle ou telle situation.

2 Un cas extrême de la revendication identitaire est celui qu'inspire le communautarisme. On entend ici ce mot comme désignant des discours et des pratiques dans lesquels les acteurs subordonnent leur représentation de l'université à l'identité sexuelle, ethnique, linguistique, religieuse qu'ils choisissent de mettre en avant dans l'organisation universitaire. Ces logiques feront dire qu'à cause de sa non appartenance à une identité sexuelle, régionale, religieuse, ethnique, ou au contraire à cause de son appartenance à une identité refusée, un acteur jugé allogène ou ennemi n'est pas qualifié pour mettre en œuvre ou critiquer des options politiques'. À l'opposé, les logiques d'identités disciplinaires, classiques à l'université, sont le fait d'acteurs qui agissent dans l'institution prioritairement comme défenseurs et représentants d'une discipline, c'est-à-dire d'une représentation de la connaissance qu'ils choisissent comme prioritaire dans leur action. D'aucune de ces deux logiques appliquées comme principe fondateur l'université, qui étymologiquement tient d'une vision universelle, ne sort indemne. 
On fait ici l'hypothèse que l'université française n'est pas encore directement touchée par la revendication communautariste même si elle en perçoit les échos, en particulier à travers certaines logiques régionalistes ou locales très poussées et peut ça et là voir certains de ses secteurs agir mus par ce moteur. Si elle se trouve, dans les années qui viennent, en proie à de tels conflits, la configuration théorique serait pour nous la même que celle qui est exposée ici. La question est en effet ici de savoir si on veut coexister avec d'autres dans l'université ou si on préfère rester entre soi qu'il s'agisse de sexe, de religion, d'origine, ou de culture professionnelle.

4 L'université est en revanche d'ores et déjà clairement traversée par des conflits qui se structurent autour d'oppositions tenant aux diverses identités professionnelles, disciplinaires, régionales, institutionnelles. Ceci en soi est totalement normal, puisqu'une organisation qui prétend à un degré minimal d'universalité ne peut exister qu'à travers un compromis passé avec ces logiques, les acteurs acceptant, dans le cadre de leur travail à l'université, dé faire passer en premier leur identité de membre d'une université. Ceci dit on peut poser que la crise ${ }^{2}$ couve et que le consensus peut se déchirer dès qu'une des identités prend le dessus de manière nette et durable. On parle ici de la crise comme du moment critique qui surgit fondamentalement d'un divorce profond et durable entre les représentations qui coexistent dans l'organisation sur cette organisation ${ }^{3}$. Ces représentations jouent un grand rôle dans le pilotage de l'organisation. Ce sont en effet elles qui permettent de définir l'objectif, dont tout étudiant en management et organisation sait qu'il est (en théorie) commun à tous les acteurs. Protégés par leur statut qui jusqu'à présent, les met à l'abri de tout licenciement, les acteurs de la vie universitaire choisissent de donner leur adhésion à telle ou telle représentation de l'action commune. En outre, depuis la loi d'orientation de 1968 ils élisent leurs dirigeants sur la base de telles représentations partagée.

5 L'ancien mode de pilotage des universités s'élaborait à partir des disciplines d'enseignement. On peut se demander s'il n'a pas vécu. Depuis la disparition des facultés et des doyens, il y a juste 35 ans et depuis l'apparition de la contractualisation et du projet d'établissement, de nouvelles logiques tendent à se substituer aux leurs. On parle désormais de la politique d'établissement à l'université, comme d'un projet auquel les acteurs peuvent s'identifier. Il est même prévu dans la réforme des statuts des universitaires que les conditions de travail, les rôles de chacun dans l'organisation, soient de plus en plus subordonnés non plus à l'identité statutaire, mais au projet d'établissement.

6 José Rose $e^{4}$ note l'importance de possibles conflits entre la légitimité politique et la légitimité académique. Les deux pouvoirs les plus anciens dans l'université sont celui des facultés et celui des disciplines. Ils datent de l'époque où l'on vivait entre soi à l'université. Ces pouvoirs et les identités qui les fondent sont mis à mal par l'intrusion des autres dans le système: par la décentralisation, l'irruption des pouvoirs locaux, la professionnalisation croissante des formations, la présidentialisation des universités et le rôle croissant des personnels non enseignants.

7 Le risque, classique en entreprise, mais plus inédit dans les universités, est que des acteurs choisissent des modes d'identification nouveaux, ce qui déstabilisera durablement certains équilibres internes.

8 Que se passe-t-il donc quand l'identité liée à l'organisation institutionnelle n'a plus le dessus le quand le projet d'établissement n'est pas ce qui structure les préoccupations et 
l'appartenance, mais qu'au contraire, les identités de certains acteurs le façonnent en fonction de leurs intérêts?

\section{Le développement des TIC et leur rôle.}

9 La croissance des budgets d'infrastructure liés aux réseaux est un des indicateurs de changement dans les modes de pilotage traditionnels. Dans une université, avant l'installation des réseaux de télécommunications, le pôle politique (la présidence) avait, pour diriger l'organisation, surtout à composer avec les « composantes » (Instituts. UFR, organisées sur des logiques de formation ou parfois encore disciplinaires: lettres, sciences, médecine technologie......). Le paysage a changé avec l'apparition de deux pôles au rôle croissant : la communication et l'informatique.

Qu'entendre par communication dans les universités? L'explosion récente des activités d'information et de communication dans les organisations a eu comme corollaire un intérêt renouvelé pour les problématiques que ces phénomènes posent. Une première série de discours, à visée fondamentalement idéologique ou industrielle, a vanté les bienfaits de la modernisation et de l'efficacité. Elle a trouvé vite ses limites. Quand on veut observer le rôle de la communication dans les universités, la question de leur «modernité » est en effet vite dépassée. Il convient de traiter de sa place concrète dans les transformations sociales de l'institution. À partir des initiatives des acteurs plus que comme l'affaire d'experts de la fonction communication. Un pôle communication s'est souvent alors développé au sein des services centraux, pour aider le fonctionnement de l'organisation et faire connaître les logiques qui l'animent.

11 Le développement des réseaux et leur rôle central dans la gestion, la communication et la croissance, la diversification, voire la survie des institutions universitaires ont vite modifié les équilibres qui existaient entre-les pôles politique, communicationnel et technologique. À l'interne, le pôle informatique s'est vite trouvé au premier plan. Les centres de calcul des années 1970 ne jouaient pas le rôle central des centres de-ressources informatiques (CRI) d'aujourd'hui, dont le rôle est désormais central dans la gestion financière et pédagogique, dans l'acquisition le maintenance et le fonctionnement d'outils informatiques (hardware et software) qui conditionnent le travail de tous les acteurs de la vie-universitaire. La coexistence dans le monde universitaire passe d'abord par les conditions matérielles d'existence, lesquelles sont gérées par ces centres. Qui délivre une adresse électronique? Qui attribue une place sur le serveur de l'université ? Qui autorise la création de listes de diffusion, de forums? Qui contrôle l'attribution des marchés de matériels informatiques et de logiciels ? Qui décide du câblage de salles ou de bureaux et assure ce câblage? Qui répartit la bande passante en fonction de priorités? Qui ? Très souvent les mêmes acteurs.

12 En termes de budget et de pouvoir de décision, les deux instances que constituent la communication et l'informatique se sont imposées dans un mode de gestion/coexistence de l'institution en forme de triangle pouvoir politique/ communication/ pôle technologique.

13 Contrôlant à l'interne deux des trois sources de pouvoir que sont la légitimité politique, le contrôle de l'information à l'interne et le contrôle de la communication avec l'extérieur ${ }^{5}$ elles ont pris un poids que les anciennes légitimités peuvent parfois difficilement égaler. 


\section{Trois tendances fortes.}

14 C'est ainsi que la coexistence dans le monde des universités est conditionnée actuellement par trois tendances fortes.

Une première tendance, à l'intérieur des organisations, réduit la communication aux seuls objets techniques. Elle ${ }^{6}$ a ainsi pour effet de renforcer le poids des ingénieurs dans le pilotage. Elle aiguise parfois les contradictions entre ces acteurs et les dirigeants traditionnellement (i.e. académiquement) ou nouvellement (i.e. politiquement) légitimes des universités. Les deux nouveaux pôles que forment la communication et l'informatique sont porteurs d'identités culturelles et professionnelles nouvelles. Ils assurent le contrôle des activités à l'université, de l'image de l'institution et de sa gestion, et établissent de nouvelles règles du jeu de la coexistence dans le monde universitaire à l'interne.

Une seconde tendance est induite par le désengagement net de l'État au profit des Régions. Elle s'accompagne d'un discours sur l'autonomie et l'initiative. Les représentants des collectivités régionales, devenues bailleurs de fonds, tiennent souvent à exister d'une manière plus présente dans les instances dirigeantes et à participer à l'élaboration des orientations universitaires. Mais cette volonté est reçue de manière ambivalente par la communauté universitaire.

Musselin et Mignot Gérard ${ }^{7}$ notent que si $74.5 \%$ des universitaires pensent que «les partenariats avec l'environnement socio économique et institutionnel sont une bonne chose pour renforcer l'assise financière des universités ». $64 \%$ ne sont pas d'accord pour que les responsables politiques et économiques soient plus impliqués dans le gouvernement des universités. Un troisième mouvement tient à l'ouverture de l'université vers l'extérieur, que ce soit en direction d'interlocuteurs régionaux distincts de l'État et des régions (les entreprises par exemple), de partenaires étrangers ou de publics lointains, que l'université peut toucher grâce aux réseaux, ou simplement de bailleurs de fonds nouveaux. Là encore, les modes de coexistence dans les instances chargées de la définition des diplômes communs, la mise en place de stratégies de publication, de prospection, relèvent de situation inédites dans l'université française classique.

18 Ces trois mouvements sont contemporains de ce que Marie Françoise Fave-Bonnet ${ }^{8}$ nomme le double mouvement de fuite en avant et tic retrait des enseignants chercheurs face au caractère incertain et morcelé de ce que devient leur identité professionnelle. Désertant leurs fonctions traditionnelles, il arrive souvent qu'ils préfèrent participer le moins possible aux structures de décision, dans lesquelles ils ne se reconnaissent plus. Ils laissent donc souvent des espaces libres à d'autres acteurs.

19 La résultante des forces exercées produit un discours qui met en valeur l'ouverture et lu coexistence. Mais, dans les faits, l'ensemble peut aussi se traduire par une morcellisation centrifuge, éventuellement animée par une alliance des mouvements liés à l'ingénierie et à la territorialisation ou encore à une "ingénierie politique » une des formes classiques, mais non la seule du pouvoir de l'ingénierie, pouvant se mesurer au poids des ingénieurs informaticiens dan les centres de décision. Le tout peut aller jusqu'à des volontés de scission. 


\section{Le cas particulier de l'université des Antilles et de la Guyane.}

20 L'université des Antilles et de la Guyane (UAG) compte actuellement 12000 étudiants. 350 enseignants (plus de 250 enseignants chercheurs) et 300 ingénieurs, techniciens et administratifs. Elle est marquée par sa situation géographique éloignée des centres de décisions ministériels (6800 kilomètres de Paris) et le caractère éclaté de ses structures tant administratives que pédagogiques : deux îles distantes entre elles de 175 kilomètres (Guadeloupe, siège de l'Université. Martinique) et une partie du continent sud-américain, la Guyane française, elle-même distante du siège de l'Université de 1535 kilomètres'.

21 Ses UFR sont éparpillées sur les trois départements et ses services communs sur l'ensemble des sites universitaires. L'UAG entretient des relations avec 3 conseils généraux. 3 conseils régionaux et 3 recteurs d'académie. Cette configuration structurelle a des conséquences sur l'organisation, notamment dans la communication et la gestion, dans l'organisation de la vie étudiante et des diplômes. Un bachelier guyanais ou guadeloupéen désireux d'étudier les lettres ou le droit doit venir s'installer en Martinique, un martiniquais ou un guyanais se destinant aux sciences exactes émigreront en Guadeloupe.

22 La cohérence de ce système trouve ses limites dès lors que des étudiants salariés s'adressent à l'université pour reprendre des études, et la contraignent d'ouvrir, à leur intention, une "Antenne lettres" en Guadeloupe ou une "Antenne sciences» en Martinique, ouvertures favorisées par les collectivités régionales.

L'université des Antilles et de la Guyane, est marquée par des identités régionales fortes et distinctes. Elle s'efforce de fonctionner depuis 1982 suivant un principe d'« interrégionalité constructive ». L'élaboration d'un projet de développement concerté vise à rationaliser le développement de l'UAG sans perdre de vue les besoins de développement de ses trois " pays ». Le poids croissant du financement des collectivités territoriales dans les structures d'enseignement supérieures peut faire craindre aux responsables universitaires la perte de contrôle du développement de l'Université. Cette éventualité constitue une raison valable pour que l'Université s'empare de cette question en proposant de formaliser ses rapports avec les collectivités territoriales. L'urgence d'entamer ce dialogue permanent avec les élus locaux est également lié à la multiplicité des interlocuteurs. L'installation de conseils régionaux dans les DOM dotés de compétences particulières en matières d'enseignement supérieur à porté le nombre des partenaires locaux de l'UAG de trois à six. Sur un plan strictement législatif, les trois régions, chacune pour leur part et sans concertation entre elles, peuvent concevoir et adopter leur projet de programmes de formations supérieures et d'activités de recherches, l'UAG doit gérer celte complexité, car son développement passe par une collaboration étroite avec ces multiples acteurs. Elle a donc une sensibilité particulièrement aiguë aux problèmes que soulève sa configuration géographique. Toute question concernant l'établissement fait l'objet d'une approche territorialement située à partir de l'un ou l'autre de ses sites. C'est au cœur de cette logique institutionnelle que s'inscrivent les logiques d'acteurs autour des activités universitaires. Au plan décisionnel, la mise en œuvre d'un développement universitaire devient souvent problématique. 
La conduite des enseignants et chercheurs, celle des personnels non enseignants, celle des étudiants dépendent de la position que chaque acteur occupe dans l'Université (catégorie, statut, fonction, service, site) mais aussi des stratégies mises en place par l'institution, voire de celles développées par ses partenaires externes en vue d'une implantation multiple ${ }^{10}$. L'UAG doit par conséquent prendre en compte plusieurs logiques :

- La motivation première tics étudiants reste de loin l'insertion professionnelle quand elle ne correspond pas simplement à une conduite que l'on qualifiera de survie. Leur action reste encore déterminée par un environnement socio-économique local qui formule ses exigences en termes d'emplois auprès de l'institution universitaire.

- La logique qui conduit l'action des personnels non enseignants peut se lire à travers les rapports qu'ils entretiennent dans et autour du travail. Leur inscription organisationnelle au sein de rétablissement et leur identité professionnelle (effectifs, statut) priment toute perspective universitaire de développement de l'enseignement supérieur et de la recherche.

- L'action des enseignants et chercheurs suit plus souvent une logique de développement externe guidée par la discipline (recherche et/ou expertise), que la logique interne attendue par l'établissement (gestion pédagogique et administrative) ${ }^{11}$. quand elle n'est pas guidée par une logique de retrait.

\section{Les CRI et les CUR : tendances à l'éclatement et ressaisissement.}

Dans un tel contexte institutionnel et géographique, la dimension stratégique des technologies de l'information et de la communication explique le rôle important que leur attribuent les responsables de l'établissement, et le développement des Centres de Ressources Informatiques. Notons d'abord que l'université en possède non pas un mais trois. Les TIC sont d'abord pour les dirigeants universitaires un enjeu du développement qui doit permettre de renforcer le gouvernement garant de l'unité institutionnelle. L'UAG compte sur les réseaux et les TIC pour la sortir de son isolement par rapport à l'hexagone et renforcer une orientation générale de modernisation et de rayonnement ${ }^{12}$, Un réseau intranet sous la responsabilité de l'administration générale lui apparaît comme un élément important du nouveau dispositif de gouvernement. ${ }^{13}$

On compte sur les CRI pour désenclaver les sites, les relier entre eux et à la métropole, pour « coordonner et mieux gérer l'utilisation des moyens modernes de communication (échanges d'information intra campus, inter campus et extra université $» .^{14}$ Les TIC doivent prendre une importance stratégique dans le développement des missions d'enseignement et de recherche et dans l'amélioration de la gestion administrative de l'établissement. L'université les voit à la fois comme vecteur de modernisation, réponse à sa dispersion géographique, et comme moyen supplémentaire de développement de ses relations internationales ${ }^{15}$.

Pour contrer les mouvements centrifuges l'Université souhaite à la fois développer le dialogue entre les trois centres de ressources informatiques existants et les faire coexister avec une commission informatique, organe statutaire chargé du schéma directeur de la politique informatique. ${ }^{16}$ 

développe sa politique informatique sur son site et la commission informatique définie par les statuts n'a jamais fonctionné. Il n'y a ni répartition des tâches, ni spécialisation des équipes (réseaux. Internet, développement spécifiques, maintenance, marchés publics...), alors que leurs moyens humains sont importants (S ingénieurs de recherche. 2 ingénieurs d'études. 4 techniciens. 1 PRAG. 1 adjoint technique).

Si pour le Comité national d'Evaluation, les CRI constituent une "avancée vers une gestion de proximité attentive aux réalité géographiques des sites» ${ }^{17}$. il existe un risque de séparatisme, qui rend cruciale la question de savoir qui « tient les manettes ». Risque renforcé par le fait que les financements de ces CRI passent par les Régions, qui ont leurs propres logiques, elles complètement territoriales. La prise en compte d'un développement cohérent avec le terrain annule finalement le problème de la coexistence puisqu'il n'y a plus de points de rencontre. La seconde structure exemplaire de cette université est celle des conseils universitaires régionaux (CUR) crées en 1999 pour permettre une décentralisation sur chacun des pôles universitaires. Leur mission consiste à organiser la vie des campus et à trouver des alternatives aux dysfonctionnements engendrés par la dispersion structurelle. Ils assurent le relais avec les services administratifs généraux de l'Université, une meilleure coordination de l'administration des UFR et des services sur site. Ils sont conçus pour améliorer la remontée des problèmes, des propositions et des solutions émanant du terrain vers l'équipe de direction. Leurs membres sont nommés et ne tiennent donc pas de légitimité d'une élection. ${ }^{18}$

Les CUR doivent assurer l'animation des pôles et une gestion de proximité (les politiques communes des composantes : accueil des étudiants, maintenance immobilière) et assurer la liaison avec le centre politique. C'est bien d'un programme visant à la coexistence et à ses conditions matérielles qu'il s'agit là. Comme la gestion administrative de l'UAG manifeste une certaine opacité structurelle, en visant l'équilibre entre gestion centrale et gestion de proximité, la nouvelle culture de l'établissement s'attaque clairement aux problèmes de communication interne. ${ }^{19}$

34 L'installation des CUR est motivée par la nécessité d'améliorer la gestion de l'UAG (finalité technique et administrative), mais surtout par celle de préserver une communauté universitaire au service de trois régions différentes (finalité politique). Ils se présentent comme le moyen de renforcer la cohérence globale de l'établissement sans entraver l'émergence d'une politique de site. Ils renforcent la structure de direction de chaque pôle, condition sine qua non du maintien de la cohésion de l'ensemble. ${ }^{20}$ Mais assurer une meilleure coordination de la politique universitaire sans empiéter sur les prérogatives des instances universitaires réglementaires, sans constituer un échelon supplémentaire, relève du défi. Assurer une meilleure coordination de lu politique universitaire en concentrant des pouvoirs, au risque de faire écran aux circuits décisionnels et administratifs tient aussi du challenge. ${ }^{21}$

En fin de compte, des instances politiques statutaires (les conseils) coexistent avec d'autres qui ne le sont pas (les CUR). Pour ne rien simplifier, les CUR menacent directement le pouvoir de l'administration générale. Sans clarification de leurs compétences et de leur articulation sur les instances réglementaires, ils sont destinés à entrer en conflit avec les composantes pédagogiques et/ou l'administration générale de l'Université. L'équilibre à trouver entre leur légitimité et celle de la coordination des pôles demeure problématique.

Communication et organisation, 24 | 2004 


\section{Les tendances scissionnistes.}

36 gestion centralisée de l'organisation universitaire, forte et continue, qui encourage les revendications d'autonomie des sites. Mais les tendances scissionnistes au sein de l'Université sont aussi exogènes. Les structures universitaires guyanaises ont généralement le sentiment d'enseignements appendices tic formations assurées en Martinique, avec des conditions matérielles très modestes. La centralisation de sa gestion administrative et pédagogique explique en partie que l'UAG ait toujours laissé aux collectivités territoriales l'initiative sur ce site. La faiblesse du budget de l'établissement est souvent invoquée pour justifier cette situation. D'une certaine manière, la radicalisation des positions guyanaises et l'action de l'État font face à une démobilisation des acteurs antillo-guyanais porteurs du projet de développement unifié de l'institution universitaire. Soit ces derniers n'appartiennent plus à l'institution (pères fondateurs), soit ils sont marginalisés par les tenants de l'évolution séparée des sites et/ou ceux du statu quo organisationnel. Ils prennent acte de la volonté d'existence d'une université guyanaise et envisagent les perspectives de développement du site en dehors de l'UAG d'autant que le débat institutionnel universitaire cache une interrogation plus politique, sur le devenir des régions d'outre-mer elles-mêmes. Le développement de renseignement et de la recherche aux Antilles Guyane doit considérer que les exécutifs régionaux sont en concurrence sur ce terrain comme des acteurs économiques. Leur poids de plus en plus déterminant dans le financement du fonctionnement de l'établissement est largement plébiscité par les responsables de l'Université. Même si ceux-ci sont opposés à une scission, que devient leur marge de manœuvre dans le pilotage de l'établissement? Que penser de la place grandissante que prennent les collectivités territoriales tant dans la définition de l'offre de formation (professionnalisation) qu'en matière d'aménagement îles campus (constructions nouvelles, réhabilitations) ? Il apparaît difficile d'écarter l'idée d'une régionalisation du développement universitaire liées aux politiques propres à chaque territoire, sauf à penser que des acteurs locaux et régionaux peu habitués à coopérer puissent impulser une dynamique nouvelle de l'offre publique d'enseignement supérieur ${ }^{22}$. Dès lors, le choix d'un mode de management des universités peut-il s'affranchir d'une inscription sur les agendas politiques ? ${ }^{23}$

De récentes et longues grèves administratives (janvier 2003 en Martinique, puis février \& mars 2003 en Guadeloupe et Guyane) ont violemment questionné l'unité de l'établissement, fes acteurs ont en effet choisi la seule base territoriale pour définir leurs revendications (en postes et titularisations) et entamer leurs négociations, ce qui a induit une rivalité fondée sur l'appartenance territoriale et non sur les appartenances professionnelles. Très à l'écoute des difficultés des personnels les plus marginalisés dans l'institution (notamment sur le site de Martinique), fort d'une bonne connaissance du terrain, un Comité Représentatif des Personnels et Usagers du Campus de Schoelcher (CREPUCS) a trouvé formalisé et utilisé lors de la grève ces éléments dans le sens qu'il souhaitait. Le comité a encouragé à la fois, un processus d'identification collective et une vision globale des enjeux. Le CREPICS «(...) développe différentes analyses visant à poursuivre, dans le dialogue et. le cas échéant, en usant des divers recours syndicaux et de mobilisation de la société civile, l'indispensable, l'incontournable restructuration de l'UAG $»^{24}$. 
39 L'éclatement de l'établissement est plus que jamais inscrit dans nombre d'agendas. Seul le pouvoir politique (universitaire et ministériel) garde comme objectif de faire coexister trois régions, trois CUR et trois CRI dans le même établissement. Nombre d'acteurs locaux cherchent plutôt un éclatement, quitte à se retrouver dans des établissements sans aucune envergure.

40 Après cinq années d'existence, les sites tendent plutôt à répondre à leurs propres demandes sans réel souci de cohérence dans une politique d'établissement.

\section{Quels types de coexistence dans les universités de demain?}

41 Une enquête de la vie universitaire ${ }^{25}$ conclut sur l'idée qu'il existerait quatre types de fonctionnement dans les universités. On peut l'entendre comme quatre modes d'organisation de la coexistence. Le premier, confédéral, règle la question de la coexistence en laissant une autonomie maximale aux composantes. Le second, fédéral, est caractérisé par une ou plusieurs disciplines ou sites qui dominent l'ensemble. Le troisième, communautaire, est celui d'établissements homogènes dans lesquels le président a un programme, mais se réserve un domaine réservé. Les conseils sont tenus par des communautés. Le quatrième, entrepreneurial est dominé par un lien fort avec les collectivités et les métiers.

On peut s'attendre à de multiples transformations de l'université, qu'elles soient liées à la volonté politique du ministère (autonomie de gestion, territorialisation de type régional, mise en concurrence...), aux transformations structurelles (poids croissant des réseaux dans tous les domaines, importance de la construction de l'espace européen de l'enseignement et de la recherche...), à l'intervention croissante d'acteurs extérieurs (régions, entreprises...), au changement d'attitudes des acteurs (mouvements consuméristes chez les étudiants, stratégies de retrait chez les enseignants chercheurs, mise en avant d'identités particulières au détriment de celle de l'établissement...).

Tous posent de manière renouvelée la question de la coexistence dans le monde de l'université. L'exemple de l'UAG montre que la gestion de proximité n'est pas une solution en soi que la mise en avant d'identités non universitaires (ici locales), ou le fait de confier l'unité de l'établissement à des logiques de pure ingénierie menacent l'unité même d'universités qui peuvent en Unir par risquer d'oublier toute référence à l'universel pour ne consacrer que le particulier.

BIBLIOGRAPHIE

L'enseignement supérieur en questions. I ${ }^{\mathrm{er}}$ colloque du Réseau d'Etude sur l'Enseignement Supérieur (RESUP). LAPSAC / Département de Sociologie - Université Victor Segalen Bordeaux 2. 16-17 mai 2002. 
Crozier, M. Friedberg. E... L'acteur et le système. Les contraintes de l'action collective. 1977. Le Seuil.

Darréon. J.-L., Filâtre D.. Les universités à l'heure de la gouvernance. Sciences de la société. 58. 2003. Presses universitaires du Mirail.

Fave-Bonnet. M.-F.. Les universitaires : une identité professionnelle incertaine. Hermès. 35. 2003. pp. 195-202.

Gabay M. Ollivier B. (dir.-). Crise et communication. Communication et organisation. 16, 2000.

Gabay M., Halatchev A., Sillam. M. « La communication universitaire à travers la presse interne et les médias externes et leur interaction : le cas de Paris 7-Denis Diderot ». Communications organisationnelles. Objets, pratiques, dispositifs. Presses Universitaires de Rennes. 2000. p. 293-310.

Gueissaz. À.. Les mondes universitaires et leur informatique. CNRS Sociologie. CNRS éditions. 1999.

Lick. R., La juste communication. La Documentation française (IDATE / Communication et Société). 1988.

Miège. B.. La société conquise par la communication. Presses Universitaires de Grenoble. 1989.

Miège. B., La société conquise par la communication - 2. La communication entre l'industrie et l'espace public. Presses Universitaires de Grenoble. 1997. Ollivier. B.. Internet, multimédia, ça change quoi dans la réalité ? INRP. 2000. 156 p.

Ollivier B., Sistemas educativos entre mâquinas e redes. Comunicaçaô \& edueaçao. IX. 25. Saô Paulo. ECA-USO. ISSN 0104-6829

Ollivier B., Les TIC à l'université des Antilles et de la Guyane. Rapport pour le Ministère de la Recherche. SDLTIC. 65 pages, dactylographié.

Pulvar. O., Stratégic(s) de communication et logique(s) d'acteurs : le cas de l'Université des Antilles et de la Guyane. Presses Universitaires duSeptentrion. 2001.

Pulvar. O., Communication et enjeux de développement à l'Université des Antilles et de la Guyane. $1^{\mathrm{er}}$ partie : Aux fondements d'une politique d'établissement (1982-1994). Espace créole. 11. GEREC-F / UAG. 2002. p. 17-27.

Pulvar. ().. Communication et enjeux de développement à l'Université des Antilles et de la Guyane. $2^{\text {nde }}$ partie : Vers une gestion moderne (1995-2003). Espace créole 12. GEREC-F /UAG (à paraître).

\section{NOTES}

1. Vladimir Jankelevitch lui-même a été jusqu'à dire en ce sens que pour un non juif critiquer la politique d'Israël était une forme d'antisémitisme. Une des caractéristiques du communautarisme est qu'il entend toutes les relations de communication comme déterminées par l'appartenance à une communauté. Réelle ou postulée (les non juifs).

2. Ollivier 2000.

3. Gabay \& Ollivier 2000

4. Darréon \& Filâtre. $2003: 46$.

5. Crozier

6. Crozier

7. Darréon \& Filâtre 2003. p. 32

8. Fave-Bonnet 2003 
9. La distance qui sépare Pointe-â-Pitre (Guadeloupe) de Schoelcher (Martinique) équivaut en kilomètres a celle qui sépare Le Havre de Paris. La distance Pointe-à-Pitre - Cayenne (AntillesGuyane) est celle de Paris à Gibraltar. L'organisation des réseaux de transport aux AntillesGuyane ne favorise pas les déplacements. Un déplacement de Martinique vers les services centraux, en Guadeloupe, coûte plus de deux cents euros De la Martinique à la Guyane, le prix peut être le même que vers Paris.

10. Pulvar 2003

11. L'université intègre plusieurs catégories de personnels enseignants avec des statuts différents et que tous ne se reconnaissent pas loin s'en faut, dans la logique

Universitaire que nous mentionnons ici.

12. UAG. Contrat quadriennal 1998-2001. p. 19

13. UAG. Contrat quadriennal 2002-2005. p. 14

14. UAG. Contrat quadriennal 1998-2001, p. 19

15. Pour une rénovai ion réfléchie du dispositif d'enseignement et de recherche en Guyane et aux Antilles. Bureau de la Présidence, mars 2001. p. 4-116

16. UAG. Contrat quadriennal 2002-2005. p. 14

17. Rapport d'évaluation de l'UAG, CNE. 2002. p. 40

18. Chaque CUR est anime par un vice-président désigné par le conseil d'administration, sur proposition du président de l'UAG. Il est membre du bureau du président qu'il représente localement II assure par la même, la coordination des politiques de pôles el la légitimité de I équipe de direction Le CUR favorise la représentation de tous les acteurs de l'enseignement supérieur, des villes universitaires el peut, en cas de besoin, inviter toute personne ou institution dont la présence s'avère nécessaire a la compréhension d'une question ou a la prise de décision Il est l'interlocuteur unique des autres partenaires institutionnels sur un pole

19. Rapport d'évaluation de l'UAG. CNE. 2002. p. 38-39.

20. Rapport dévaluation de l'UAG. CNE. 2002. p. 33 ; UAG. Contrat QUADRIENNAL 1998-2001. p.17-18: UAG. Contrat quadriennal 2002-2005. p. 3.

21. UAG. Contrat quadriennal 1998-2001. p. 17 ; Rapport d'évaluation de l'UAG. CNE. 2002. p. 115.

22. Filâtre. 2002

23. La participation des collectivités territoriales aux ressources de l'établissement $(12,5 \%$ contre $5.2 \%$ en moyenne nationale) a permis d'améliorer sa capacité d'accueil L'intervention des collectivités territoriales apparaît aux responsables universitaires comme un encouragement pour l'Université à jouer pleinement son rôle d'acteur du développement régional (Contrat quadriennal 1998-2001. Introduction, p.2). L'UAG définit des critères de délocalisation en fonction notamment $\mathrm{du}$ soutien des collectivités locales (UAG. Contrat quadriennal 2002-2005.p. 3). La question peut se lire a la lumière des débats actuels autour de l'évolution institutionnelle des DOM et de la décentralisation.

24. Livre blanc du pôle Martinique de l'Université des Antilles et de la Guyane. CREPUCS, janvier 2003, p. 2.

25. Darréon et Filâtre. 2003 


\section{AUTEURS}

\section{OLIVIER PULVAR}

Université Antilles - Guyane 